\title{
To Study the Effect of the Concentration of Carbon on Ultraviolet and Visible Light Photo Catalytic Activity and Characterization of Carbon Doped $\mathrm{TiO}_{2}$
}

\author{
Rajkumar $\mathbf{R}^{1 *}$ and Nisha Singh ${ }^{2}$
}

${ }^{1}$ Department of Chemical Engineering, Bipin Tripathi Kumaon Institute of Technology, Dwarahat, Almora, Uttrakhand-263653, India ${ }^{2}$ Department of Chemical Engineering, R.N. College of Engineering and Technology, Panipat, Haryana-132103, India

\begin{abstract}
Photo catalytic degradation of pollutants in water or air using titanium dioxide has been considered one of the efficient methods but it shows poor absorption of visible light and requires ultraviolet light for its activation. Which increase the cost of system, So that we moved toward the doping of non-metal in $\mathrm{TiO}_{2}$ which gave good result in visible and UV light, carbon doped $\mathrm{TiO}_{2}$ is prepared using air heating method at lowest temperature and minimum time by taking glucose and ethanol as carbon source. The experimental results showed that carbon was successfully doped into $\mathrm{TiO}_{2}$ The physical properties of carbon doped $\mathrm{TiO}_{2}$ were studies by XRD, TG-TGA and UV spectroscopy which showed that the photo catalytic efficiency increase in UV and visible lights by creation of oxygen vacancies and $\mathrm{Ti}^{3+}$ in $\mathrm{C}-\mathrm{TiO}_{2}$ catalyst. UV spectroscopy results showed the optical response of $\mathrm{TiO}_{2}$ was shifted from UV to the visible light region.
\end{abstract}

Keywords: C-doped $\mathrm{TiO}_{2}$ nano particles; Photocatalysis; $\mathrm{TiO}_{2}$; Doping with non-metals; Preparation; Low temperature

\section{Introduction}

Semiconductor titanium dioxide $\left(\mathrm{TiO}_{2}\right)$ is a promising photocatalyst for air purification, water disinfection, hazardous waste remediation and water purification [1]. It exists as three different polymorphs; anatase, rutile and brookite [2]. It is typically an n-type semiconductor due to oxygen deficiency [3]. The band gap is $3.2 \mathrm{eV}$ for anatase and $3.0 \mathrm{eV}$ for rutile $[4,5]$. Unfortunately, because of its large band gap of $3.2 \mathrm{eV}$, it can be activated only under UV light irradiation of wavelength $(<387 \mathrm{~nm})$. However, UV light constitutes only a small fraction (about 3-5\%) of the solar spectrum; it will have a positive effect for improving the photo catalytic efficiency of $\mathrm{TiO}_{2}$ by shifting its optical response to the visible range. Hence scientists are exploring methods to modify $\mathrm{TiO}_{2}$ to absorb visible light. For example, carbondoped $\mathrm{TiO} 2$ was found to be effective for water splitting under visible light. Most of these investigations had been made to convert the $\mathrm{TiO}_{2}$ so that its absorption power shift from the UV to the visible region by doping of $\mathrm{TiO}_{2}$ with transition metals such as $\mathrm{Cr}, \mathrm{Fe}, \mathrm{Mn}, \mathrm{V}$ [6-11], etc. However the photo catalytic activity of transition metals doping is poor because of their thermal instability [10]. The non-metal doping has been considered for about ten years [11] as a best way to change the photoabsorption properties of $\mathrm{TiO}_{2}$ for using of solar light with applications in photocatalysis and photo electrochemistry [12-15]. Due to doping, the dopants of non-metals in the lattice might affect the band edges or introduce impurity states in the band gaps of the system [16,17]. Carbon-doped titania with high surface area $\left(204 \mathrm{~m}^{2} / \mathrm{g}\right)$ was prepared by temperature programmed carbonization of $\mathrm{K}$-contained anatase titania under a flow of cyclohexane at temperatures between 450 and $500^{\circ} \mathrm{C}$. This carbon-doped titania has much better photo catalytic activity for gas-phase photo-oxidation of benzene under irradiation of artificial solar light than pure titania. Obviously, these non-metals doping in $\mathrm{TiO}_{2}$ were all synthesized at high temperature. So it is still a challenge to prepare non-metal doped $\mathrm{TiO}_{2}$ at a low temperature, especially for the energy-saving production of visible-light driven photo catalyst in a large scale for pollutants removal. They prepared carbon doped $\mathrm{TiO}_{2}$ by hydrothermal treatment at $160^{\circ} \mathrm{C}$ which displayed stronger absorption in both UV and visible range. These papers gave me the knowledge to develop a low-temperature method to prepare carbon-doped $\mathrm{TiO}_{2}$, by using ethanol and titanium oxide Degussa (P25) as the carbon and $\mathrm{TiO}_{2}$ sources, respectively. The carbon doping could take place at the same time under a new method by using as heating medium except water vapor treatment at $180^{\circ} \mathrm{C}$ for $4 \mathrm{~h}$ at constant heating in hot air oven. To the best of our knowledge, this is the lowest temperature to prepare carbon-doped $\mathrm{TiO}_{2}$ using air as heating medium. It is found that the resulting carbon-doped $\mathrm{TiO}_{2}$ by this new method exhibits much higher photo catalytic activity than the undoped counterpart and Degussa P25 on the degradation of phenol under visible and UV light. The focus of this work is on the determination of the true concentration of raw, exfoliated HiPCO SWNTs in the supernatant phase. A UV-visible absorption-based approach is suggested for a direct measurement of the SWNT and the surfactant concentration in the supernatant. UV-visible absorbance spectra of SWNTs-surfactant dispersions and surfactants alone reveal that the intensity of a certain peak, attributed to the $\pi$-plasmon resonance absorption, is unaffected by the presence of most surfactants. A calibration plot is then made by monitoring the intensity of the peak as a function of the true concentration of the exfoliated SWNTs. Thus, we are able to determine the unknown concentration of surfactant-dispersed HiPCO SWNTs in the supernatant solution, simply by measuring its optical absorbance. Moreover, we can now calculate the surfactant efficiency in dispersing SWNTs. Cryogenic-transmission electron microscopy and thermo

*Corresponding author: Rajkumar R, Department of Chemical Engineering, Bipin Tripathi Kumaon Institute of Technology, Dwarahat, Almora, Uttrakhand-263653, India, Tel: 91-7830131877; E-mail: ranvir523@gmail.com

Received October 17, 2014; Accepted January 06, 2015; Published January 15 2015

Citation: Rajkumar R, Singh N (2015) To Study the Effect of the Concentration of Carbon on Ultraviolet and Visible Light Photo Catalytic Activity and Characterization of Carbon Doped $\mathrm{TiO}_{2}$. J Nanomed Nanotechnol 6: 260. doi:10.4172/21577439.1000260

Copyright: @ 2015 Rajkumar R, et al. This is an open-access article distributed under the terms of the Creative Commons Attribution License, which permits unrestricted use, distribution, and reproduction in any medium, provided the original author and source are credited. 
gravimetric analysis techniques are used for the characterization of these dispersions and to complement the UV-visible measurements.

\section{Experimental}

\section{Sample preparation}

Carbon doped $\mathrm{TiO}_{2}$ nanoparticles were prepared by air heating method by taking $4 \mathrm{~g}$ of $\mathrm{TiO}_{2}$ and $40 \mathrm{ml}$ ethanol. The mixture of ethanol and $\mathrm{TiO}_{2}$ was constantly stirred by magnetic stirrer for $10 \mathrm{~min}$ and then the heated for $4 \mathrm{~h}$ at $180^{\circ} \mathrm{C}$ in hot air oven. The catalyst was kept in dissector for cooling to obtain white powder of carbon doped $\mathrm{TiO}_{2}$. For calcinations half of catalyst was kept in muffle furnace for 1 hour at $350^{\circ} \mathrm{C}$. Similarly this method is used for making of different concentration of ethanol C- $\mathrm{TiO}_{2}$ at are $92.20 \%$ ethanol $\mathrm{TiO}_{2}$ at $94.04 \%$, ethanol $\mathrm{TiO}_{2}, 95.17 \%$ ethanol $\mathrm{TiO}_{2}, 95.94 \%$ ethanol $\mathrm{TiO}_{2}$ etc.

\section{Characterization of $\mathrm{C}$-doped $\mathrm{TiO}_{2}$ nano particles}

$\mathrm{X}$-ray powder diffraction (XRD) analysis was performed using Goniometer=PW3050/60 X-ray diffract meter $(\mathrm{CuKa} \mathrm{k}=0.154 \mathrm{~nm})$. The working voltage and current of the X-ray tube were $45 \mathrm{kV}$ and 40 $\mathrm{mA}$, AVarian Cary 100 Scan UV-visible system equipped with a lab sphere diffused reflectance accessory was used to obtain the absorbance spectra of the catalysts over a range of 200-600 $\mathrm{nm}$. Lab sphere USRS99-010 was employed as a absorbance standard.

\section{Photocatalytic degradation experiments}

The photocatalytic degradation experiments of methyl orange by the $\mathrm{TiO}_{2}$ photocatalysts were performed under visible light irradiation. The photocatalytic degradation was monitored by use of a UV-Vis spectrometer at $\lambda \max =468 \mathrm{~nm}$. In all experiments a $100 \mathrm{mg}$ of catalyst was suspended in $100 \mathrm{ml}$ of $5 \mathrm{ppm}$ methyl orange solution in a conical flask. The flask was wrapped with aluminum foil then placed in the dark for an hour for adsorption to take place. The system was then irradiated with visible light from a lamp that was fixed in the middle of the system and $12 \mathrm{~cm}$ above the surface of the solution. To detect changes in concentration, aliquots of methyl orange $(5 \mathrm{~mL})$ were taken after every 30 minutes and centrifuged. The absorbance of the clean solution was measured by a UV-Vis spectrometer.

\section{Results and Discussion}

\section{Crystal characterization}

To characterize the crystalline structure of the carbon doped and undoped $\mathrm{TiO}_{2}$ samples, for that XRD patterns in Figures 1 and 2 were obtained. The pattern of undoped $\mathrm{TiO}_{2}$ showed the presence of anatase and bookie However, only anatase is present in the XRD pattern of C-doped $\mathrm{TiO}_{2}$ indicate the doping of carbon prohibit the formation of brookite. It can be also found that the crystal size of anatase decrease with the carbon doping by comparing the (101) peak of anatase in Figures 1 and 2. This decrease on crystal size can be attributed to dissimilar boundaries created by carbon doping. Meanwhile, the significant decrease on the intensity of XRD pattern of $\mathrm{TiO}_{2}$. The crystal size decrease can be attributed to dissimilar boundaries created by carbon doping, because of the significant decrease on the intensity of XRD pattern of $\mathrm{TiO}_{2}$. The diffraction peaks of XRD of carbon doped $\mathrm{TiO}_{2}$ were not observed which showed that carbon was highly dispersed on $\mathrm{TiO}_{2}$ or XRD was not sensitive enough to detect such minor changes to $\mathrm{TiO}_{2}$.

The recently started R\&D activity of growth and characterization of NLO single crystals has been continued in view of (i) growing good quality crystals (ii) growing big size crystals suitable for device applications related to photonics, (iii) to enhance the efficiency of second harmonic generation (SHG) by using different dopants and functional groups, (iv) to search new NLO materials and grow their single crystals, ( $\mathrm{v}$ ) to evaluate their crystalline perfection by highresolution XRD and (vi) other studies like powder XRD, FTIR, UV-Vis. etc. In this endeavor and with the help of various collaborators working in this upcoming area we have achieved several important R\&D results leading to twenty one articles in leading SCI journals and one article in non SCI journal. Some of these important results are briefly described in Figures 1 and 2.

If a sample contains anatase and rutile, the mass fraction of anatase $\left(\mathrm{W}_{\mathrm{A}}\right)$ and futile $\left(\mathrm{W}_{\mathrm{R}}\right)$ can be calculated from the equations 1 and 2:

$$
W_{A}=\frac{K_{A} A_{A}}{K_{A} A_{A}+A_{R}}
$$

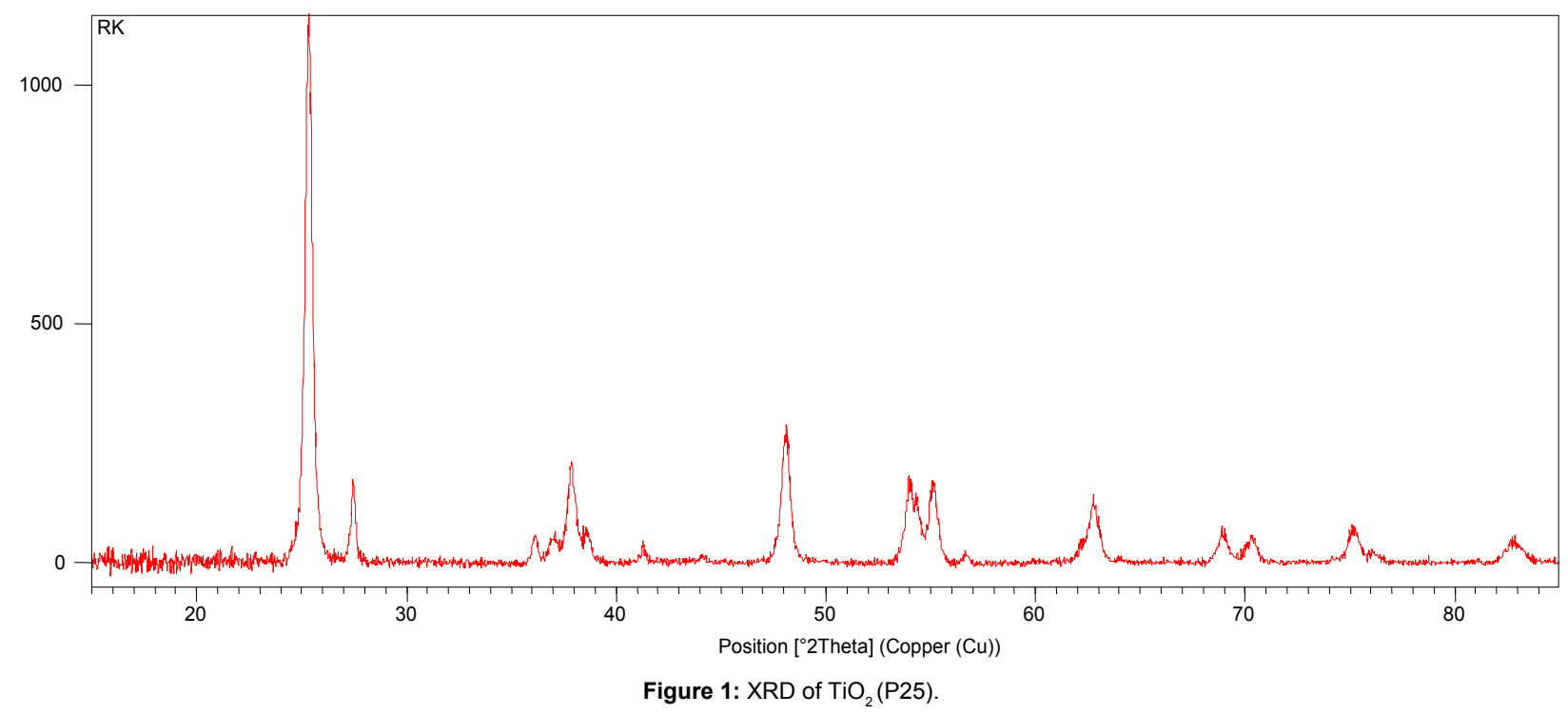




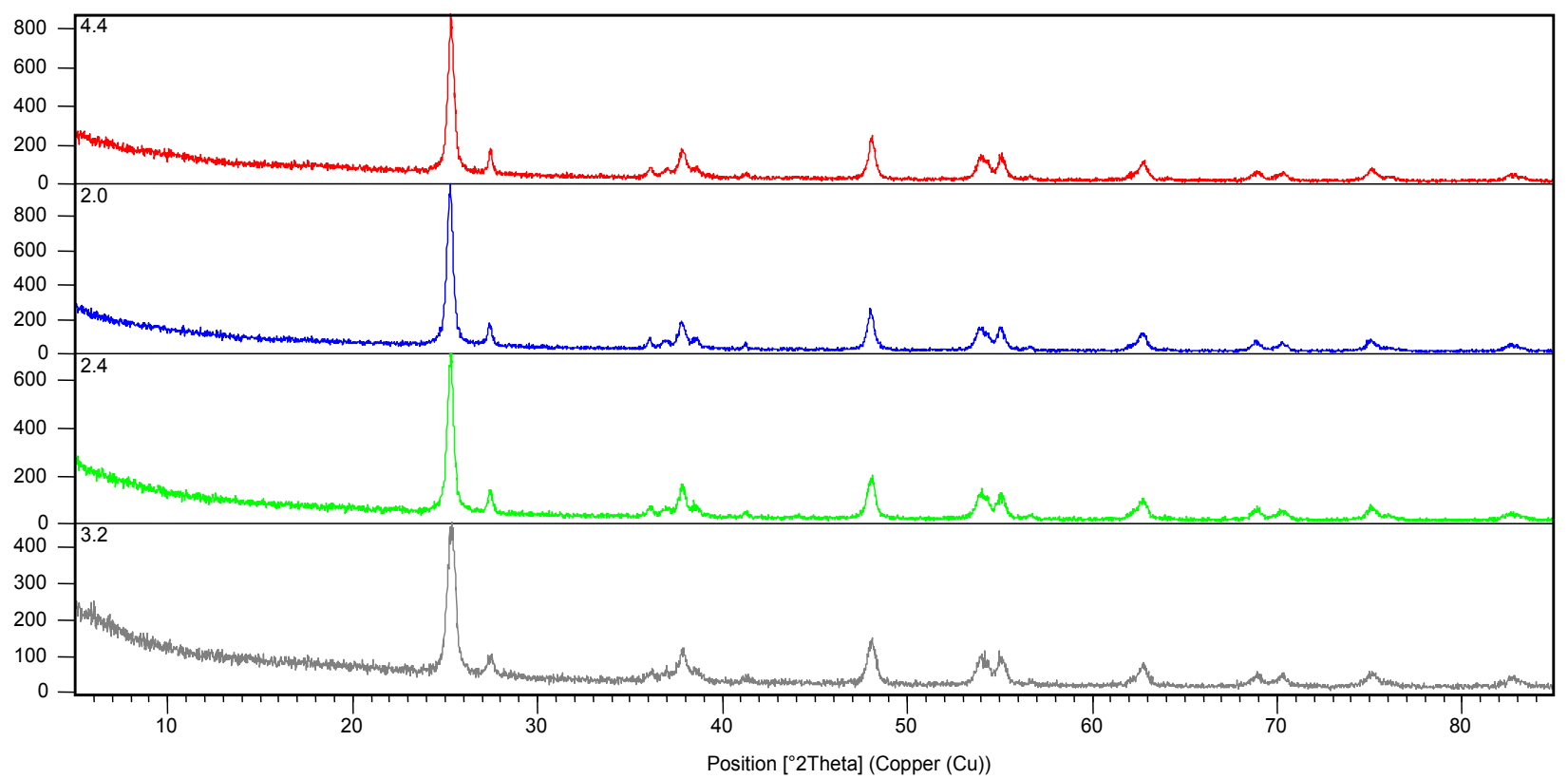

Figure 2: Comparison of $\mathrm{XRD}$ of different concentration ethanol doped $\mathrm{TiO}_{2}$.

\begin{tabular}{|c|c|}
\hline Sample & Size(nm) \\
\hline $\mathrm{TiO}_{2}$ & 48.22 \\
\hline $88.75 \%$ ethanol and $4 \mathrm{~g} \mathrm{TiO}_{2}$ & 41.74 \\
\hline $92.20 \%$ ethanol and $4 \mathrm{~g} \mathrm{TiO}_{2}$ & 43.68 \\
\hline $94.04 \%$ ethanol and $4 \mathrm{~g} \mathrm{TiO}_{2}$ & 60.20 \\
\hline $95.17 \%$ ethanol and $4 \mathrm{~g} \mathrm{TiO}_{2}$ & 61.01 \\
\hline $95.94 \%$ ethanol and $4 \mathrm{~g} \mathrm{TiO}_{2}$ & 62.50 \\
\hline
\end{tabular}

Table 1: Size comparisons between $\mathrm{TiO}_{2}$ and its carbon $\mathrm{TiO}_{2}$.

$$
W_{R}=\frac{A_{A}}{K_{A} A_{A}+A_{R}}
$$

Where $A_{A}$ and $A_{R}$ are the integrated intensity of the anatase (101) and rutile (110) peaks, respectively, $W_{A}$ and $W_{R}$ represents the mass fraction of anatase and rutile, respectively, and $\mathrm{K}_{\mathrm{A}}$ is a coefficient and its value is 0.886 . The average crystallite sizes of anatase were determined as follows:

$$
D=\frac{0.9 \lambda}{\beta \cos \theta}
$$

Where $\lambda$ is the wavelength of characteristics X-ray applied and $\beta$ is the half-value width of anatase (101) peak obtained by XRD and is X-ray diffraction angle. According to the Scherrer equation using the data of each different phase after correcting the instrumental broadening in Table 1.

\section{UV-vis diffuse reflectance spectra}

UV vis absorption of undoped $\mathrm{TiO}_{2}$ 88.75\%, 92.20\%, 95.94\% ethanol doped $\mathrm{TiO}_{2}$ are shown in Figures 3 and 4 respectively. Carbon-doped $\mathrm{TiO}_{2}$ sample absorbs more light in the range of 400-450. Obviously, the optical absorption edges of these non-metal doped samples shift to the lower energy region compare to the undoped $\mathrm{TiO}_{2}$. This showed that our method is effective to extend the absorption to visible light range. A plot of the $(\mathrm{ahv})^{1 / 2}$ versus the energy of absorbed light affords the band gap energy approximately 2.90 and $3.1 \mathrm{eV}$ for the carbon-doped $\mathrm{TiO}_{2}$ and pure $\mathrm{TiO}_{2}$, respectively. This result obviously reveals the band gap of the carbon-doped $\mathrm{TiO}_{2}$ is narrower than that of the pure $\mathrm{TiO}_{2}$.

\section{TGA analysis}

To see the variation in weight of $\mathrm{C}-\mathrm{TiO}_{2}$, TGA measurements were carried out. The results are shown in Figure 5. As expected, the $\mathrm{C}-\mathrm{TiO}_{2}$ powders can be oxidized by oxygen in the air at an elevated temperature of From $300^{\circ} \mathrm{C}$ the total weight increase is about $10 \%$. If some amount of carbon were oxidized, the weight increase would be $31.25 \%$ as calculated from the reaction: $\mathrm{C}-\mathrm{TiO}_{2}+4 \mathrm{O}_{2}-\mathrm{TiO}_{2}+\mathrm{CO}_{2}$. The amounts of residual carbon and are dependent on the hot air oven temperature.

\section{Photocatalytic activity}

The effect of catalyst concentration on photo catalytic degradation efficiency of $\mathrm{TiO}_{2}$ was investigated by keeping all other experimental parameter constant. It is observed that there is steady increase in the degradation efficiency up to $5 \mathrm{~g} / \mathrm{l}$ of the catalyst beyond which the degradation efficiency decrease. Within the range of catalyst dose from 2.5 to $5.0 \mathrm{~g} / \mathrm{l}$, observed phenomena enhancement in degradation may be due to an increase in available absorption and catalyst sites on the surface of carbon doped $\mathrm{TiO}_{2}$. A further increase in concentration of catalyst however, may cause light scattering, blocking of UV light and particles of agglomeration which decrease the number of active sites. 


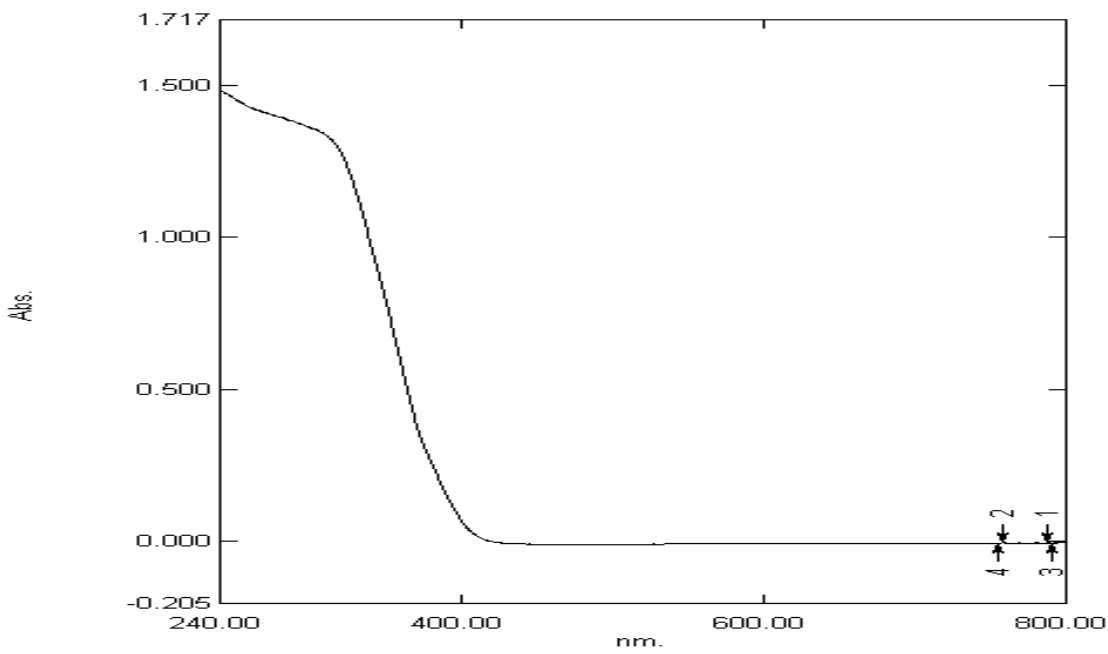

Figure 3: UV-Vis Spectrophotometer of pure $\mathrm{TiO}_{2}$.

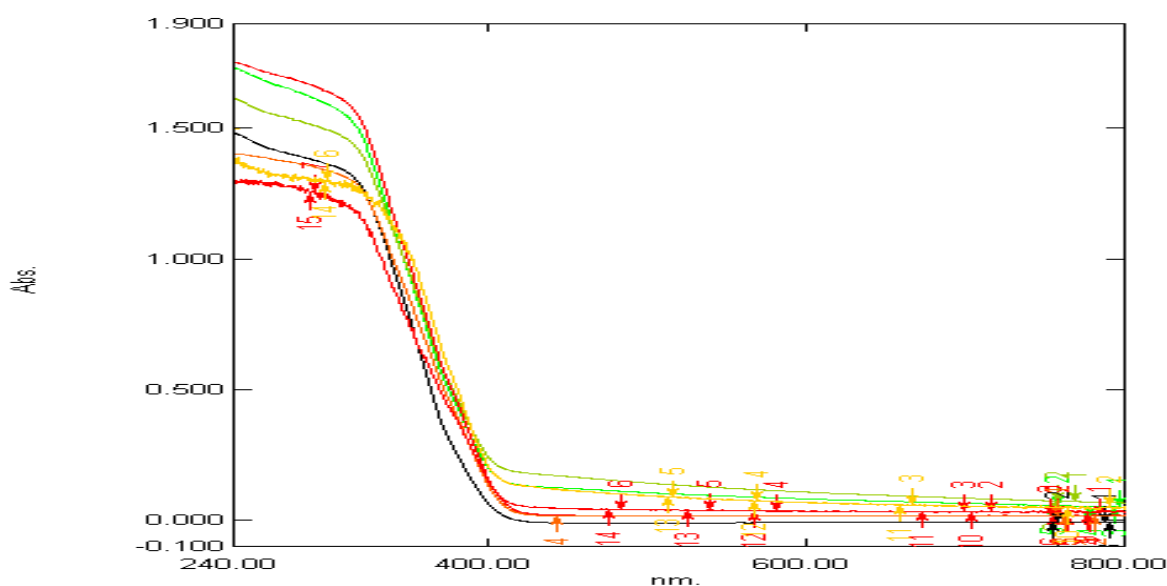

Figure 4: Comparison of UV-Vis Spectrophotometer of different concentration ethanol doped $\mathrm{TiO}_{2}$.

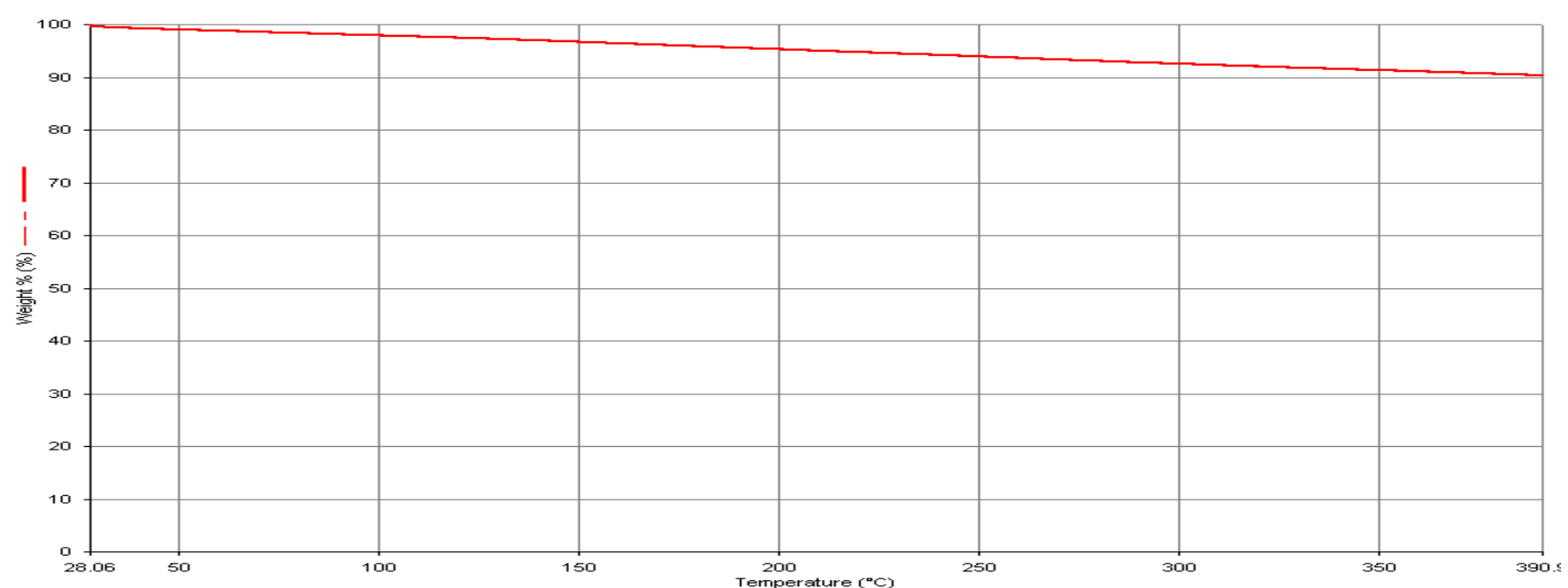

Figure 5: TGA graph of carbon doped $\mathrm{TiO}_{2}$. 
Citation: Rajkumar R, Singh N (2015) To Study the Effect of the Concentration of Carbon on Ultraviolet and Visible Light Photo Catalytic Activity and Characterization of Carbon Doped $\mathrm{TiO}_{2}$. J Nanomed Nanotechnol 6: 260. doi:10.4172/2157-7439.1000260

Page 5 of 7

The result indicates that an optimized catalyst concentration $5 \mathrm{~g} / \mathrm{l}$ is necessary for enhancing the degradation efficiency, as shown in Figures 6 and 7.

Figures 8 and 9 shows the photocatalitic degradation curves of 100ppm over the P25, 88.75\%, 92.20\%, 95.94\% ethanol doped

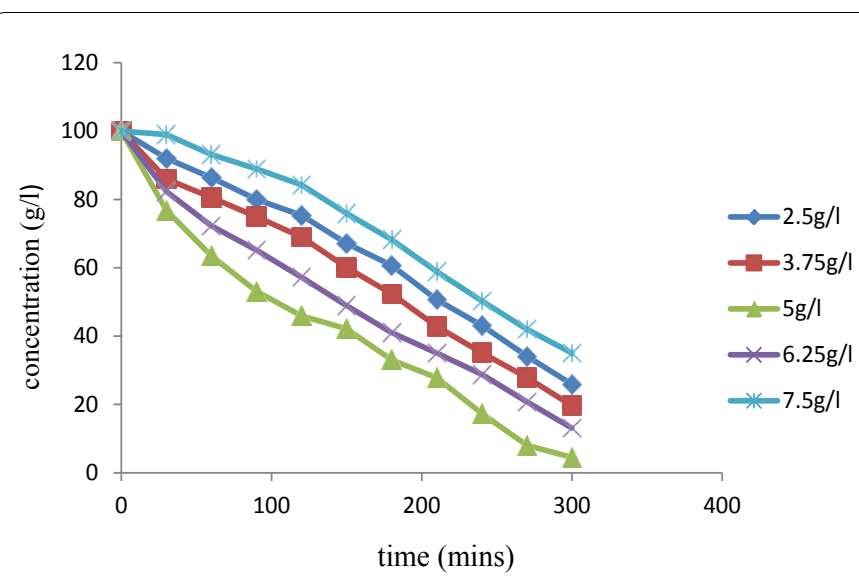

Figure 6: Comparison of degradation of phenol with loading concentration of $\mathrm{TiO}_{2}$ photo catalyst in UV light.

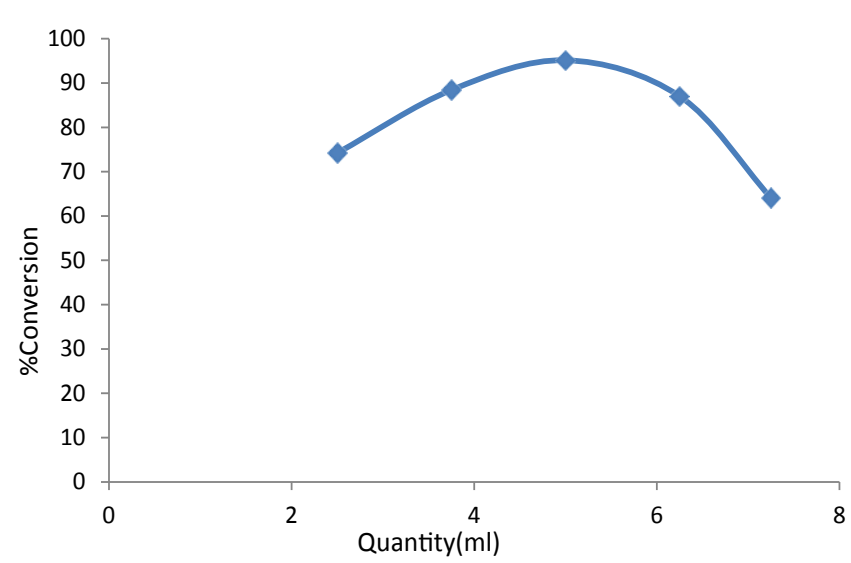

Figure 7: Comparison of \% conversion between different quantities of catalyst loading of $\mathrm{TiO}_{2}$.

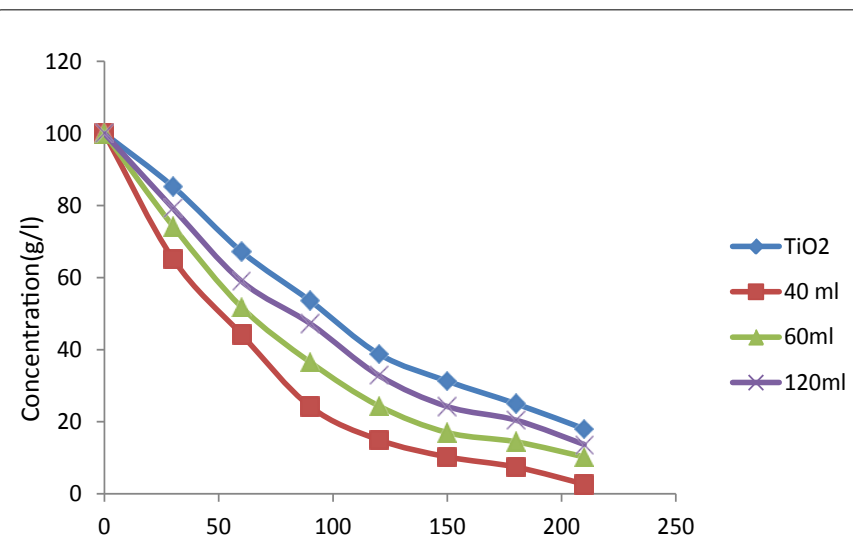

Figure 8: Comparison of degradation of phenol with different concentration ethanol doped $\mathrm{TiO}_{2}$ and undoped $\mathrm{TiO}_{2}$ of photo catalyst in UV light.

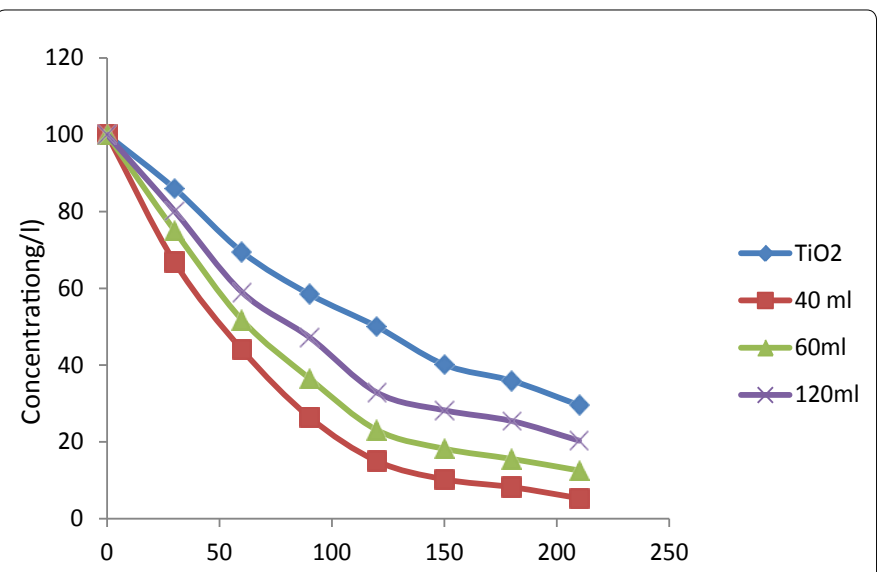

Figure 9: Comparison of degradation of phenol with different concentration ethanol doped $\mathrm{TiO}_{2}$ and undoped $\mathrm{TiO}_{2}$ of photo catalyst in sunlight.

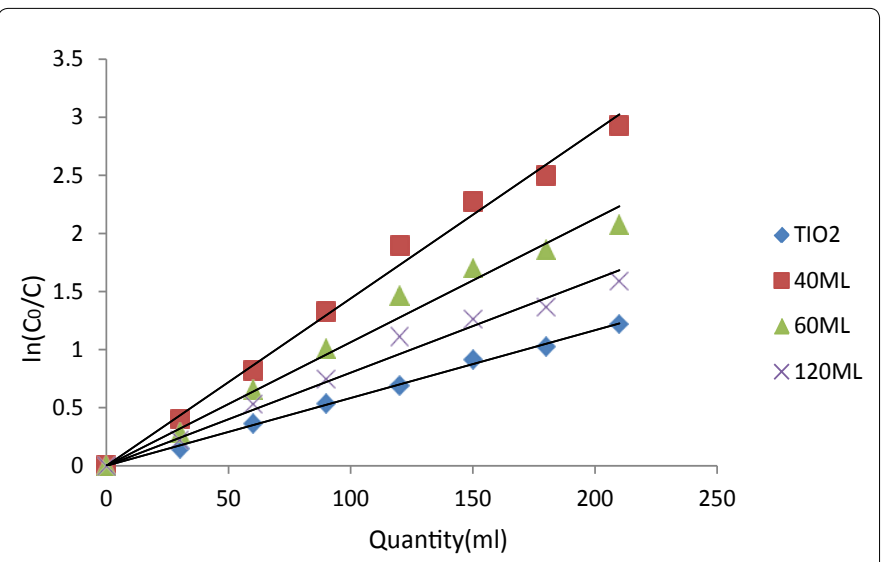

Figure 10: Comparion of pure $\mathrm{TiO}_{2}, 88.75 \% .92 .20 \%$ and $95.94 \%$ under UV light.

$\mathrm{TiO}_{2}$ photocatalysts with different carbon concentration and got maximum degradation near about $97 \%$ of 100 ppm phenol solution at $88.75 \%$ ethanol doped $\mathrm{TiO}_{2}$ under $\mathrm{UV} / \mathrm{V}$ is light. The photocatalytic degradation of $100 \mathrm{ppm}$ phenol solution reaction times is first order with $\mathrm{K}$ from 0.0144 to $0.0058 \mathrm{~min}^{\wedge}-1$ in concentration range of $88.75 \%$ $-95.94 \%$ ethanol in $\mathrm{TiO}_{2}$ as confirmed by the linear transformation of $\ln (\mathrm{C} / \mathrm{C}) \sim \mathrm{t}$ shown in Figures 10 and 11. Apparent rate constants in UV light were obtained with various catalyst samples with different ethanol concentration from Figure 12 and graph shows that $\mathrm{K}$ decrease with increase in the initial concentration of ethanol and maximum $\mathrm{K}$ is 0.0144 for $88.75 \%$ ethanol doped in $\mathrm{TiO}_{2}$ because XRD and UV result shows that $88.75 \% \mathrm{C}-\mathrm{TiO}_{2}$ have minimum partical size $(41.74 \mathrm{~nm})$ and maximum shift in visible region $(450 \mathrm{~nm})$. When ethanol concentration added greater than $88.75 \%$ that decrease photocatalytically active sites on the surface of catalyst particals and reduing the intreaction of photons with these sites.which can reduced the photocatalytic activity.

The photocatalytic degradation of 100ppm phenol solution reaction times is psedue first order with $\mathrm{K}$ from 0.0154 to 0.0078 $\min ^{\wedge}-1$ in concentration range of $88.75 \%-95.94 \%$ ethanol in $\mathrm{TiO}_{2}$ as confirmed by the linear transformation of $\ln \left(\mathrm{C}_{\mathrm{o}} / \mathrm{C}\right) \sim \mathrm{t}$ shown in Figure 11 . We analyze that when we increase concentration of ethanol in $\mathrm{TiO}_{2}$ degradation of phenol under solar light increases because rate constant increase and varies in between 0.0158 to $0.0078 \min ^{\wedge}-1$. As shown in Figure 13 the rate constant in solar light is higher than that of 


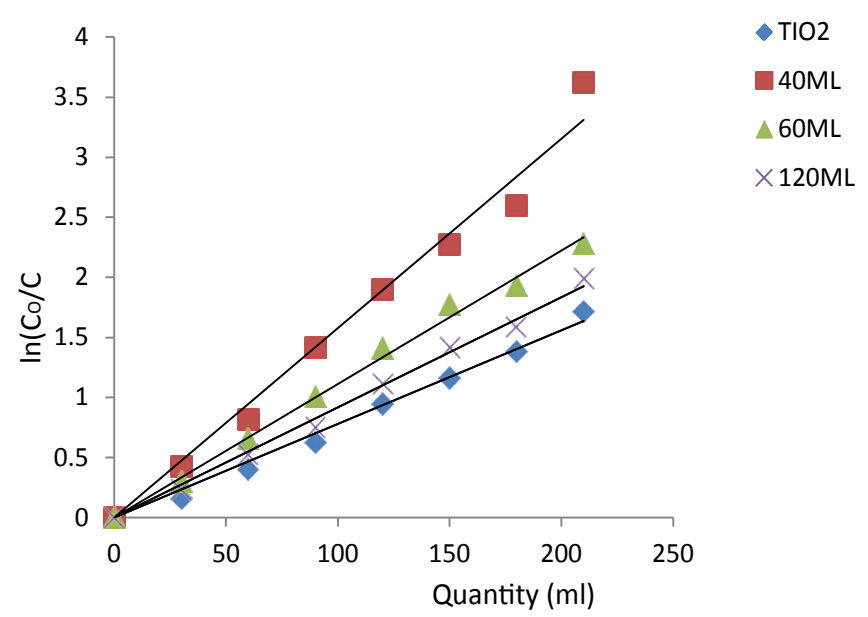

Figure 11: Comparion of pure $\mathrm{TiO}_{2}, 88.75 \% .92 .20 \%$ and $95.94 \%$ under solar light.

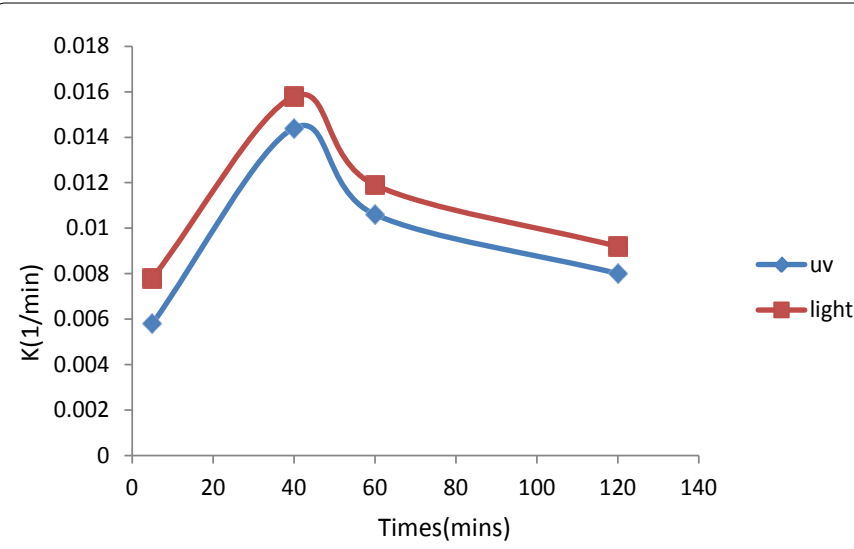

Figure 12: Graph between rate constant and quantity of ethanol for UV light and sunlight.

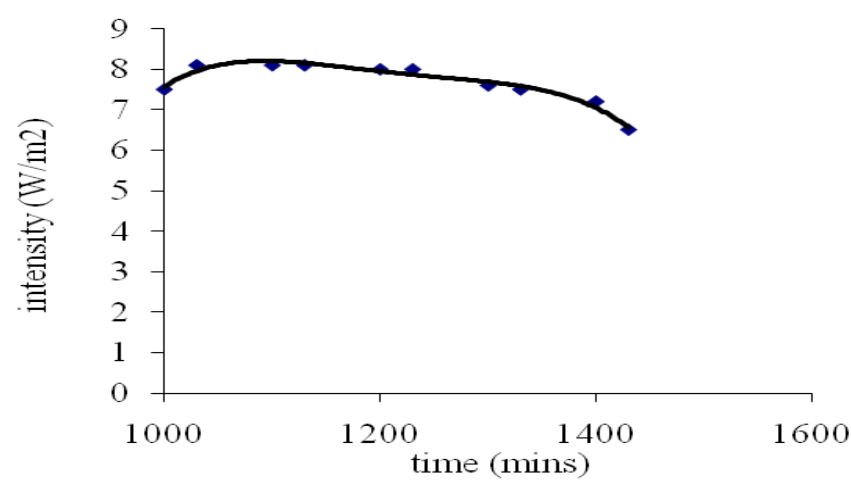

Figure 13: Graph between intensity of light and time.

UV light so degradation of phenol solution in solar light is higher than UV light. The increased photoactivity of carbon-doped $\mathrm{TiO}_{2}$ may be associated with more-complex phenomena than the simple presence of the impurity atoms, such as, for instance, concomitant changes in the stoichiometry of the sample. According to laser flash photolysis experiments, 15 the holes formed upon visible light excitation are less reactive than those formed upon $\mathrm{UV}$ excitation in pure $\mathrm{TiO}_{2}$, because they are trapped at midgap levels induced by carbon doping. Lesser mobility is expected only for the holes but not for the electrons, which are excited into the conduction band. This means that carbon-doped systems exhibit decreased direct oxidation ability. On the other hand, reduction properties or indirect oxidation properties (via reactions with surface intermediates of water oxidation or oxygen reduction) are much improved upon irradiation with visible light, also because the holes trapped at the carbon doping site do not serve as effective charge recombination centers [15] and carbon doping favors the formation of oxygen vacancies. This feature makes nonmetal doping of $\mathrm{TiO}_{2}$ much more appealing than transition-metal doping. Analysis of the computed electronic structure shows that carbon doping in anatase results in a series of localized occupied states in the band gap whose density and nature are dependent on the interaction with the oxide matrix, the dopant concentration, and the presence of oxygen vacancies. It was observed that rate constant of $40 \mathrm{ml}$ ethanol doped have higher in UV light than visible. Under UV irradiation, the best degradation of phenol was achieved in the presence of $88.75 \%$ ethanol in $\mathrm{TiO}_{2}$ and, it was observed that at relatively high ethanol concentrations, nanoparticles agglomerated which resulted in decreased surface area and the corresponding decrease photocatalytic activity in sun light. Therefore, increasing the dopant concentration has two opposite effects on the photocatalytic activity of the carbon doped $\mathrm{TiO}_{2}$ catalysts; increasing light absorption capacity and decreasing surface area. The photocatalytic activity depends on which one of these is the dominant factor. A lesser amount of surface hydroxyl groups was observed in the case of undoped $\mathrm{TiO}_{2}$ compared with the ethanol doped $\mathrm{TiO}_{2}$. This could be one of the reasons for the lower activity of the undoped $\mathrm{TiO}_{2}$ catalysts. Most of the non metal doped $\mathrm{TiO}_{2}$ samples absorb in the visible region was no direct correlation between the light absorption capacity of the carbon doped $\mathrm{TiO}_{2}$ catalysts and the rate of phenol solution degradation. It should be noted that the degradation rates in the presence of carbon doped $\mathrm{TiO}_{2}$ catalysts under $\mathrm{UV}$ were in general less than that of the undoped $\mathrm{TiO}_{2}$. Recombination of photo generated electrons and holes are one of the most significant factors that deteriorate the catalytic properties.

\section{Intensity of sunlight}

This was clearly explained in Figure 13.

\section{UV-Vis absorption of the $\mathrm{TiO}_{2}$ nanoparticles}

The UV-visible absorption spectra of the C-doped. $\mathrm{TiO}_{2}$ were shifted by between $7 \mathrm{~nm}$ to $36 \mathrm{~nm}$ depending on the doping level as shown in Figure 14. The highest shift of $36 \mathrm{~nm}$ was due to $\mathrm{TiO}_{2}$ doped with the lowest concentration of dopant $(0.093 \mathrm{M}$ glucose $)$ while the lowest shift of about $7 \mathrm{~nm}$ was recorded for the sample doped with the highest concentration of dopant $(0.278 \mathrm{M}$ glucose). The absorbance increased with a decrease in the loading of the glucose, indicating that a higher concentration of the glucose led to a smaller particle size. The band gap was therefore increased as particle size decreased.

\section{Conclusions}

A visible-light-active carbon $\mathrm{TiO}_{2}$ nano particles acts as a photocatalyst was prepared by a new approach through carbon doping by using ethanol as carbon source at a lowest temperature by heating with air. Carbon-doped $\mathrm{TiO}_{2}$ possessed a high surface area and exhibited obvious absorption in the visible light range than P25 and undoped counterpart. It was found that carbon-doped $\mathrm{TiO}_{2}$ showed significantly higher absorbance in visible than $\mathrm{P} 25$ and undoped $\mathrm{TiO}_{2}$ under visible 
Citation: Rajkumar R, Singh N (2015) To Study the Effect of the Concentration of Carbon on Ultraviolet and Visible Light Photo Catalytic Activity and Characterization of Carbon Doped $\mathrm{TiO}_{2}$. J Nanomed Nanotechnol 6: 260. doi:10.4172/2157-7439.1000260

Page 7 of 7

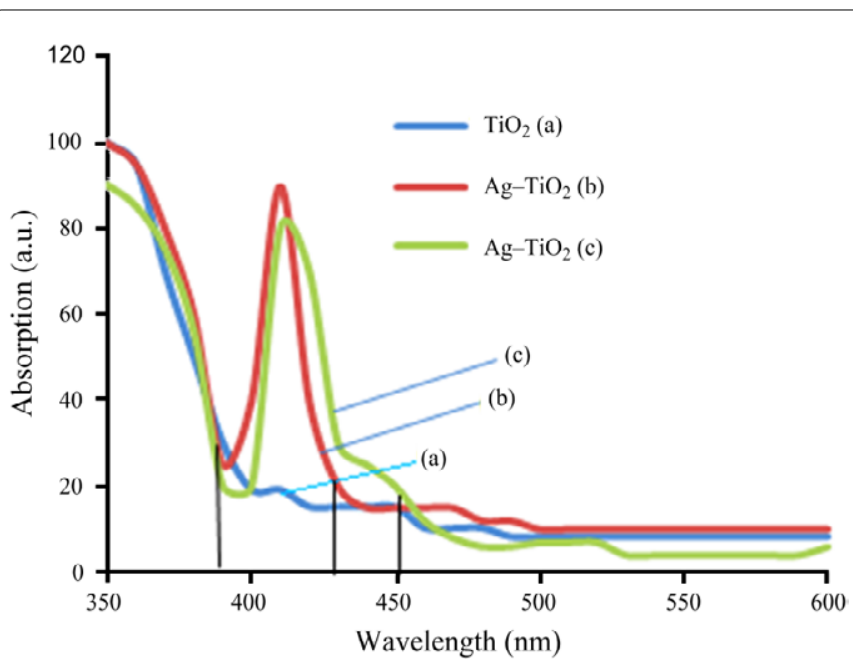

Figure 14: Diffuse reflectance spectra of undoped $\mathrm{TiO}_{2}$ and carbon doped $\mathrm{TiO}_{2}$ prepared with different loadings of glucose.

light irradiation. Our method is potential for industrial production of visible-light driven photocatalyst because of its convenience and energy-saving.

\section{References}

1. Hoffmann MR, Martin ST, Choi W, Bahnemannt DW (1995) Environmental Applications of Semiconductor Photocatalysis. Chem Rev 95: 69-96.

2. Nolan NT, Seery MK, Pillai SC (2009) Spectroscopic Investigation of the Anatase-to-Rutile Transformation of Sol- Gel-Synthesized $\mathrm{TiO}_{2}$ Photocatalysts. J Phy Chem C 113: 16151-16157.

3. Wisitsoraat A, Tuantranont A, Comini E, Sberveglieri G, Wlodarski W (2009) Characterization of n-type and p-type semiconductor gas sensors based on $\mathrm{NiOx}$ doped TiO2 thin films. Thin Solid Films 517: 2775-2780.

4. Asahi R, Taga Y, Mannstadt W, Freeman AJ (2000) Electronic and optical properties of anatase TiO2. Physical Review B 61: 7459-7465.
5. Koelsch M, Cassaignon S, Ta Thanh Minh C, Guillemoles JF, Jolivet JP (2004) Electrochemical comparative study of titania (anatase, brookite and rutile) nanoparticles synthesized in aqueous medium. Thin Solid Films 451: 86-92.

6. Yamashita H, Ichihashi Y, Takeuchi M, Kishiguchi S, Anpo M (1999) Characterization of metal ion-implanted titanium oxide photocatalysts operating under visible light irradiation. J Synchrotron Radiat 6: 451-452.

7. Klosek S, Raftery D (2001) Visible Light Driven V-Doped TiO2 Photocatalyst and Its Photooxidation of Ethanol. J Phys Chem B 105: 2815-2819.

8. Anpo M, Takeuchi M (2003) The design and development of highly reactive titanium oxide photocatalysts operating under visible light irradiation. J Catal 216: 505-516.

9. Ghosh AK, Maruska HP (1977) Photoelectrolysis of Water in Sunlight with Sensitized Semiconductor Electrodes. J Electrochem Soc 124: 1516-1522.

10. Choi WY, Termin A, Hoffmann MR (1994) Role of Metal-lon Dopants in Quantum-Sized TiO2 - Correlation between Photoreactivity and Charge-Carrier Recombination Dynamics. J Phys Chem 98: 13669-13679.

11. Asahi R, Morikawa T, Ohwaki T, Aoki K, Taga $Y$ (2001) Visible-light photocatalysis in nitrogen-doped titanium oxides. Science 293: 269-271.

12. Di Valentin C, Finazzi E, Pacchioni G, Selloni A, Livraghi S, et al. (2008) Density Functional Theory and Electron Paramagnetic Resonance Study on the Effect of $\mathrm{N}-\mathrm{F}$ Codoping of TiO2. Chem Mater 20: 3706-3714.

13. Di Valentin C, Pacchioni G, Onishi H, Kudo A (2009) Cr/Sb co-doped TiO2 from first principles calculations. Chem Phys Lett 469: 166-171.

14. Zhu W, Quu X, lancu V, Chen XQ, Pan H, et al. (2009) Band gap narrowing of titanium oxide semiconductors by noncompensated anion-cation codoping for enhanced visible-light photoactivity. Phys Rev Lett 103: 226401.

15. Czoska AM, Livraghi S, Paganini MC, Giamello E, Di Valentin C, et al. (2011) The nitrogen-boron paramagnetic center in visible light sensitized N-B codoped $\mathrm{TiO}(2)$. Experimental and theoretical characterization. Phys Chem Chem Phys 13: 136-143.

16. Li YZ, Hwang DS, Lee NH, Kim NSJ (2005) Synthesis and characterization of carbon-doped titania as an artificial solar light sensitive photocatalyst. Chem Phys Lett 404: 25-29.

17. Sakthivel S, Kisch H (2003) Daylight photocatalysis by carbon-modified titanium dioxide. Angew Chem Int Ed Engl 42: 4908-4911. 